\title{
ON THE DYNAMICS OF CONTINUOUS DISTRIBUTIONS OF DISLOCATIONS
}

\author{
$\mathrm{BY}$
}

ERNST BINZ, GÜNTER SCHWARZ. AND JAN WENZELBURGER

Dept. of Mathematics and Computer Science, Univ. of Mannheim, D-68131 Mannheim, Germany

\begin{abstract}
For materials with continuous distributions of dislocations a configuration space which unifies the continuum theory of defects with classical elasticity is given. Weak equations of motion are derived from the principle of virtual work. Using the Helmholtz decomposition theorem, this yields a coupled system of equations for the dynamics of dislocations and classical elasticity.
\end{abstract}

1. Introduction. The classical approach in continuum theory of defects is based on the idea that dislocations of a crystalline body $M$ can be described by torsions of flat connections on the body manifold $M$, cf. [11] and references therein. In a different approach, [20] and [25] used the notion of a torsion to describe defects in material bodies. More recently, it was shown in [27] that dislocation densities may be described by exact $\mathbb{R}^{3}$-valued differential two-forms $d \gamma \in \Omega^{2}\left(M ; \mathbb{R}^{3}\right)$. This observation is based on the fact that the Burgers vector for a given dislocation density of a material body $M$ computes as the integral of an $\mathbb{R}^{3}$-valued two-form $d \gamma$ over a bounded surface $S \subset M$.

The purpose of this paper is to put forward a first step towards the dynamics of a crystalline material using the calculus of differential forms. To this end, we utilize the description of dislocation densities by exact $\mathbb{R}^{3}$-valued two-forms $d \gamma$. On the other hand, we use the fact that the space $E\left(M ; \mathbb{R}^{3}\right)$ of all embeddings of a reference body $M$ into $\mathbb{R}^{3}$ constitutes the appropriate configuration space in classical elasticity. The differentials of these embeddings are the essential quantities for the constitutive behavior of an elastic material, cf. [3]. In classical terms, embeddings are also called configurations and their differentials are deformation gradients. We focus on the internal structure of a crystalline material with continuous distributions of dislocations in the sense of [11], often referred to as a continuized crystal. Following [28], we define a generalized configuration space for a material with dislocations $\mathcal{V}\left(M ; \mathbb{R}^{3}\right)$ as a submanifold of $\Omega^{1}\left(M ; \mathbb{R}^{3}\right)$ such that the gradient part $d j_{\gamma} \in \Omega^{1}\left(M ; \mathbb{R}^{3}\right)$ of each generalized configuration $\gamma \in \mathcal{V}\left(M ; \mathbb{R}^{3}\right)$ stems from

Received December 8, 1997.

2000 Mathematics Subject Classification. Primary 53C80, 58A10, 58A14.

We would like to thank an anonymous referee for helpful comments.

Current address of the third author: University of Bielefeld, Department of Economics, P.O. Box 100 131, D-33501 Bielefeld, Germany. 
an embedding $j_{\gamma} \in E\left(M ; \mathbb{R}^{3}\right)$. In classical terms, $d j_{\gamma}$ is precisely the deformation gradient of an actual configuration $j_{\gamma}$ of the system, whereas $d \gamma$ corresponds to the dislocation density associated with this configuration. The constitutive behavior of materials with continuous distributions of dislocations is encoded in a virtual work functional $F$ on the configuration space $\mathcal{V}\left(M ; \mathbb{R}^{3}\right)$. D'Alembert's principle of virtual work then allows us to derive weak equations of motion for generalized configurations $\gamma$.

The key result, which allows us to incorporate the description of dislocations into the framework of elasticity, is the Helmholtz decomposition theorem for differential forms. It claims that any differential form $\gamma \in \Omega^{1}\left(M ; \mathbb{R}^{3}\right)$ can be uniquely decomposed into a gradient part $d u_{\gamma}$ and a divergence-free part $\beta_{\gamma}$, such that $\gamma=d u_{\gamma}+\beta_{\gamma}$. Since $d \circ d=0$, any dislocation density $d \gamma \in \Omega^{2}\left(M ; \mathbb{R}^{3}\right)$ of a material is completely determined by the divergence-free part $\beta_{\gamma}$ of $\gamma$. This implies that dislocation densities are encoded in the space of all divergence-free one-forms $\mathcal{D}\left(M ; \mathbb{R}^{3}\right)$. A generalized configuration $\gamma \in \mathcal{V}\left(M ; \mathbb{R}^{3}\right)$ splits by construction uniquely into an elastic or gradient part $d j_{\gamma}$, where $j_{\gamma} \in E\left(M ; \mathbb{R}^{3}\right)$ is an embedding, and into a so-called plastic component $\beta_{\gamma}$ describing the dislocation density. The configuration space is constructed in such a way that the description of all possible dislocation densities is included.

We use d'Alembert's principle of virtual work to derive weak equations of motion for generalized configurations $\gamma$. If $\gamma(\cdot):[0, T] \rightarrow \mathcal{V}\left(M ; \mathbb{R}^{3}\right)$ is a (weak) solution of these dynamical equations, then by the Helmholtz decomposition theorem, this curve splits into $\gamma(t)=d j_{\gamma}(t)+\beta_{\gamma}(t)$ for each $t \in[0, T]$. That is, the solution curve can be uniquely decomposed into a curve $d j_{\gamma}(\cdot):[0, T] \rightarrow d E\left(M ; \mathbb{R}^{3}\right)$ of deformation gradients and a curve $\beta_{\gamma}(\cdot):[0, T] \rightarrow \mathcal{D}\left(M ; \mathbb{R}^{3}\right)$ of divergence-free one-forms describing the dislocation densities. The Helmholtz decomposition may be applied on the level of the weak equations of motions as well. Hence, we obtain a coupled system of partial differential equations for $\left(d j_{\gamma}(t), \beta_{\gamma}(t)\right) \in d E\left(M ; \mathbb{R}^{3}\right) \times \mathcal{D}\left(M ; \mathbb{R}^{3}\right)$ which determines the evolution of the elastic components $d j_{\gamma}$ of a generalized configuration $\gamma$ as well as its plastic components $\beta_{\gamma}$. The equations extend those obtained in [9], in providing an additional differential equation for the fluxes of dislocation densities. In particular, in the absence of dislocations, the equations for the elastic components are just the well-known equations in classical elasticity. This approach thus generalizes classical elasticity.

To complete a description of the dynamics of dislocations, one has to stipulate constitutive equations for a material on physical grounds. In the presence of dislocations, dissipation and irreversibility, which usually is accompanied by plastic deformations, have to be taken into account. This can be incorporated into this framework by a suitable choice of the virtual work functional $F$, because the work functional in our approach need not stem from a potential. In particular, $F$ could parametrically depend on thermodynamic quantities such as a temperature field. The thermodynamic equations for these quantities then could well be from an irreversible type. Since the discussion of these effects is beyond the scope of this work, we refer the reader to [19]. For the sake of simplicity, we also neglect effects of external fields such as gravitation and (external) electro-magnetism which, in principle, could be included, cf. [6]. External mechanical forces are incorporated through boundary conditions on the actual configuration of the material. 
2. Differential forms. Let the body manifold of the mechanical system be represented by a compact 3-dimensional Riemannian manifold with boundary which is embeddable into the physical space $\mathbb{R}^{3}$. An $\mathbb{R}^{3}$-valued differential form $\omega \in \Omega^{k}\left(M ; \mathbb{R}^{3}\right)$ is a smooth skew-symmetric fiberwise $k$-linear map $\omega$ from the tangent bundle $T M$ to $\mathbb{R}^{3}$. Differential forms can be identified with skew-symmetric two-point tensors of type $(1, k)$ on $M$, which are well-known objects in continuum mechanics, cf. [16]. In particular, the deformation gradient and the first Piola-Kirchoff stress tensor are $\mathbb{R}^{3}$-valued one-forms on $M$, i.e., some $\omega \in \Omega^{1}\left(M ; \mathbb{R}^{3}\right)$. Also, the moment stress tensor can be transformed into an $\mathbb{R}^{3}$-valued differential form on $M$. Likewise, displacement fields of $M$ and force fields are elements in $\Omega^{0}\left(M ; \mathbb{R}^{3}\right)$ which, by definition, is equal to $C^{\infty}\left(M ; \mathbb{R}^{3}\right)$.

Let $E_{1}, E_{2}, E_{3} \in \Gamma(T M)$ be an orthonormal frame, that is, a triple of vector fields that are orthonormal with respect to the Riemannian metric $g$ on $M$, and let $\langle\cdot, \cdot\rangle_{\mathbb{R}^{3}}$ denote the standard scalar product on $\mathbb{R}^{3}$. Then the space $\Omega^{1}\left(M ; \mathbb{R}^{3}\right)$ is equipped with a fibre metric defined by

$$
\langle\omega, \eta\rangle:=\sum_{l=1}^{3}\left\langle\omega\left(E_{l}\right), \eta\left(E_{l}\right)\right\rangle_{\mathbb{R}^{3}}, \quad \omega, \eta \in \Omega^{1}\left(M ; \mathbb{R}^{3}\right) .
$$

The product (1) depends only on the metric $g$ but not on the choice of the frame. In terms of a basis $\left(e_{1}, e_{2}, e_{3}\right)$ on $\mathbb{R}^{3}$ and coordinates $\left(\theta^{1}, \theta^{2}, \theta^{3}\right)$ on $M,(1)$ corresponds to the contraction of two-point tensors. That is, if $\omega=\sum_{L, l} \omega_{l}^{L} d \theta^{l} e_{L}$ and $\eta=\sum_{L, l} \eta_{l}^{L} d \theta^{l} e_{L}$, then

$$
\langle\omega, \eta\rangle=\sum_{L, l=1}^{3} \omega_{l}^{L} \eta_{l}^{L}
$$

With the help of the Riemannian volume element $\mu$ induced by $g$, the space $\Omega^{1}\left(M ; \mathbb{R}^{3}\right)$ is endowed with an $L^{2}$-product $\mathcal{G}$, given by

$$
\mathcal{G}(\omega, \eta):=\int_{M}\langle\omega, \eta\rangle \mu, \quad \omega, \eta \in \Omega^{1}\left(M ; \mathbb{R}^{3}\right) .
$$

On 0 -forms, i.e., functions, the corresponding $L^{2}$-product $\mathcal{G}$ is just the usual one:

$$
\mathcal{G}(u, v):=\int_{M}\langle u, v\rangle_{\mathbb{R}^{3}} \mu, \quad u, v \in C^{\infty}\left(M ; \mathbb{R}^{3}\right) .
$$

Let $\omega \in \Omega^{1}\left(M ; \mathbb{R}^{3}\right)$ be an $\mathbb{R}^{3}$ valued one-form and $X \in \Gamma(T M)$ be a vector field. Then

$$
D[\omega(X)](Y)
$$

denotes the directional derivative of $\omega(X) \in C^{\infty}\left(M ; \mathbb{R}^{3}\right)$ in the direction of the vector field $Y \in \Gamma(T M)$. The exterior derivative $d: \Omega^{1}\left(M ; \mathbb{R}^{3}\right) \rightarrow \Omega^{2}\left(M ; \mathbb{R}^{3}\right)$ is given by

$$
d \omega(X, Y)=D[\omega(Y)](X)-D[\omega(X)](Y)-\omega([X, Y]), \quad X, Y \in \Gamma(T M),
$$

where $[X, Y]$ denotes the Lie bracket of vector fields. The exterior derivative of a function $u \in C^{\infty}\left(M ; \mathbb{R}^{3}\right)$ is the one-form $d u \in \Omega^{1}\left(M ; \mathbb{R}^{3}\right)$ defined by the directional derivative, i.e., $d u(X):=D[u](X)$. The co-differential $\delta: \Omega^{1}\left(M ; \mathbb{R}^{3}\right) \rightarrow C^{\infty}\left(M ; \mathbb{R}^{3}\right)$ is the formal 
adjoint of the exterior derivative $d: C^{\infty}\left(M ; \mathbb{R}^{3}\right) \rightarrow \Omega^{1}\left(M ; \mathbb{R}^{3}\right)$ with respect to the $L^{2}$ product $\mathcal{G}$. In terms of an orthonormal frame, it reads

$$
\delta \omega:=-\sum_{l=1}^{3} D\left[\omega\left(E_{l}\right)\right]\left(E_{l}\right) .
$$

In classical tensor notation, $d$ corresponds to the gradient and $\delta$ to the divergence of a tensor field. Let $\mathcal{N}$ denote the outward pointing unit normal field on the boundary $\partial M$ of $M$. A differential one-form $\omega$ is said to be parallel to $\partial M$ if its normal component vanishes, that is, $\omega(\mathcal{N})=0$. We denote the space of all divergence-free and parallel one-forms by

$$
\mathcal{D}\left(M ; \mathbb{R}^{3}\right):=\left\{\omega \in \Omega^{1}\left(M ; \mathbb{R}^{3}\right) \mid \delta \omega=0 \text { and } \omega(\mathcal{N})=0\right\}
$$

and the space of all gradients of $\mathbb{R}^{3}$-valued functions by $d C^{\infty}\left(M ; \mathbb{R}^{3}\right)$. The Helmholtz decomposition for $\mathbb{R}^{3}$-valued one-forms is then stated as follows. For a general version and a proof see [22].

Theorem 2.1 (Helmholtz Decomposition). Let $M$ be a compact, oriented Riemannian manifold with boundary. For any $\omega \in \Omega^{1}\left(M ; \mathbb{R}^{3}\right)$ there exist $u \in C^{\infty}\left(M ; \mathbb{R}^{3}\right)$ and $\beta \in \mathcal{D}\left(M ; \mathbb{R}^{3}\right)$ such that $\omega=d u+\beta$. Moreover, $d u$ and $\beta$ are mutually $L^{2}$-orthogonal with respect to the inner product $(2)$. That is, $\Omega^{1}\left(M ; \mathbb{R}^{3}\right)$ allows for an $L^{2}$-orthogonal direct decomposition

$$
\Omega^{1}\left(M ; \mathbb{R}^{3}\right)=d C^{\infty}\left(M ; \mathbb{R}^{3}\right) \oplus \mathcal{D}\left(M ; \mathbb{R}^{3}\right)
$$

3. Dislocation densities. The classical approach to elastic solids with dislocations, see e.g. [11], typically characterizes the interior structure by a triple of linearly independent vector fields on $M$

$$
Z_{1}, Z_{2}, Z_{3} \in \Gamma(T M)
$$

Physically, this frame, sometimes called a material frame, describes lattice vectors of a continuized ideal crystal. Let $e_{1}, e_{2}, e_{3}$ denote the standard basis of $\mathbb{R}^{3}$. By assumption on $M$, for any choice of a frame (6), there exists a unique map $\gamma: T M \rightarrow \mathbb{R}^{3}$ such that

$$
\gamma_{p}\left(Z_{i}(p)\right)=e_{l}, \quad l=1,2,3 \quad \forall p \in M
$$

Mathematically, $\gamma$ is an $\mathbb{R}^{3}$-valued one-form on $M$, say $\gamma \in \Omega^{1}\left(M ; \mathbb{R}^{3}\right)$, which is fiberwise one-to-one. The set of all these one-forms is denoted by

$$
\mathcal{I}\left(M ; \mathbb{R}^{3}\right):=\left\{\gamma \in \Omega^{1}\left(M ; \mathbb{R}^{3}\right) \mid \gamma_{p}: T_{p} M \rightarrow \mathbb{R}^{3} \text { is one-to-one, } p \in M\right\} .
$$

Consider a fixed $\gamma \in \mathcal{I}\left(M ; \mathbb{R}^{3}\right)$. Then each $\gamma(X) \in C^{\infty}\left(M ; \mathbb{R}^{3}\right), X \in \Gamma(T M)$ is a smooth function whose directional derivatives (4) exist. A connection $\nabla[\gamma]$ on $T M$ associated with $\gamma \in \mathcal{I}\left(M ; \mathbb{R}^{3}\right)$ is defined by

$$
\nabla[\gamma]_{Y} X=\gamma^{-1} D[\gamma(X)](Y), \quad X, Y \in \Gamma(T M)
$$


The connection $\nabla[\gamma]$ corresponds precisely to the material connection of the elastic body with dislocations, cf. $[20,25]$. In local coordinates $\left(\theta^{1}, \theta^{2}, \theta^{3}\right)$ on $M$, the corresponding Christoffel symbols can be defined by

$$
\nabla[\gamma]_{\frac{\partial}{\partial \theta^{k}}} \frac{\partial}{\partial \theta^{l}}=\sum_{n=1}^{3} \Gamma_{k l}^{n} \frac{\partial}{\partial \theta^{n}}
$$

(see [23, p. 6-2]), such that

$$
\Gamma_{k l}^{n}=\sum_{L=1}^{3}\left(\gamma^{-1}\right)_{L}^{n} \partial_{k} \gamma_{l}^{L} .
$$

It is easy to verify that the curvature of $\nabla[\gamma]$ vanishes, i.e., the connection defined by (8) is flat. In fact, the relation between the space of all connections on $M$ and the differential forms $\gamma \in \mathcal{I}\left(M ; \mathbb{R}^{3}\right)$ is even closer: if $M$ is simply connected, then for any flat connection $\widetilde{\nabla}$ on $T M$, there is some $\gamma \in \mathcal{I}\left(M ; \mathbb{R}^{3}\right)$ with $\widetilde{\nabla}=\nabla[\gamma]$, cf. [27]. The torsion of an arbitrary connection $\nabla$ is defined by

$$
T^{\nabla}(X, Y)=\nabla_{Y} X-\nabla_{X} Y-[X, Y] \quad \forall X, Y \in \Gamma(T M) .
$$

In particular, if $T[\gamma]$ denotes the torsion of $\nabla[\gamma]$, it follows from the definition of the exterior derivative $d: \Omega^{1}\left(M ; \mathbb{R}^{3}\right) \rightarrow \Omega^{2}\left(M ; \mathbb{R}^{3}\right)$ that

$$
d \gamma(X, Y)=\gamma(T[\gamma](X, Y)), \quad X, Y \in \Gamma(T M)
$$

The classical approach uses torsions of flat connection for describing dislocation densities or material inhomogeneities associated with the material frame (6). Let $\gamma \in$ $\mathcal{I}\left(M ; \mathbb{R}^{3}\right)$ be the corresponding one-form determined by $(7)$. Since $\gamma$ is fibrewise one-toone, it is meaningful to consider the inverse $\gamma_{p}^{-1}: \mathbb{R}^{3} \rightarrow T_{p} M$ at each point $p \in M$. From (9) we infer that $T[\gamma]=\gamma^{-1} d \gamma$. Therefore, the dislocation density $T[\gamma]$ of a dislocated material can equally well be measured by the exterior derivative of $\gamma$. In particular,

$$
d \gamma=0 \Leftrightarrow T[\gamma]=0,
$$

implying that the material is defect-free if and only if $\gamma$ is closed, i.e., $d \gamma=0$. Moreover, it can be shown that for any exact two-form $d \omega \in \Omega^{2}\left(M ; \mathbb{R}^{3}\right)$, there exists an injective $\gamma \in \mathcal{I}\left(M ; \mathbb{R}^{3}\right)$ such that $d \omega=d \gamma$. For this reason, each exact $\mathbb{R}^{3}$-valued two-form $d \omega$ describes a dislocation density on $M$, cf. [28, Theorem 4.1]. Summarizing we have

Theorem 3.1. The internal structure of a material, described by a frame $\left(Z_{1}, Z_{2}, Z_{3}\right) \in$ $\Gamma(T M)$ is completely determined by a one-form $\gamma \in \mathcal{I}\left(M ; \mathbb{R}^{3}\right)$. In particular, the dislocation density $T$ is completely characterized by the exterior derivative $d \gamma \in \Omega^{2}\left(M ; \mathbb{R}^{3}\right)$ via $T[\gamma]=\gamma^{-1} d \gamma$. If $S \subset M$ is an arbitrary surface, the Burgers vector $b$ corresponding to the chosen frame computes as the integral

$$
b=\int_{S} d \gamma
$$

In view of the above theorem, it is natural to refer to the two-form $d \gamma \in \Omega^{2}\left(M ; \mathbb{R}^{3}\right)$ as the dislocation density. It is crucial to observe that each $\gamma \in \mathcal{I}\left(M ; \mathbb{R}^{3}\right)$ uniquely splits 
into

$$
\gamma=d u_{\gamma}+\beta_{\gamma}, \quad \text { where } u_{\gamma} \in C^{\infty}\left(M ; \mathbb{R}^{3}\right), \beta_{\gamma} \in \mathcal{D}\left(M ; \mathbb{R}^{3}\right) .
$$

This is an immediate consequence of the Helmholtz decomposition, Theorem 2.1. Since $d^{2}=0$, only the divergence-free component $\beta_{\gamma} \in \mathcal{D}\left(M ; \mathbb{R}^{3}\right)$ of $\gamma$ contributes to the dislocation density, $d \gamma \in \Omega^{2}\left(M ; \mathbb{R}^{3}\right)$, i.e., $d \gamma=d \beta_{\gamma}$. The classical conservation law for dislocation densities (cf. [15, p. 135]) reads $d^{2} \beta_{\gamma}=0$ and hence is automatically satisfied. Summarizing we have:

Corollary 3.1. Let $\left(Z_{1}, Z_{2}, Z_{3}\right) \in \Gamma(T M)$ describe the interior structure on $M$. Then the dislocation density of the material is uniquely described by the nonexact component $\beta_{\gamma} \in \mathcal{D}\left(M ; \mathbb{R}^{3}\right)$ of the one-form $\gamma \in \mathcal{I}\left(M ; \mathbb{R}^{3}\right)$ characterizing the material.

4. The kinematics of dislocations. Let $E\left(M ; \mathbb{R}^{3}\right)$ denote the space of all smooth embeddings of the body manifold $M$ into the physical space $\mathbb{R}^{3}$. Since the tangent bundle of $\mathbb{R}^{3}$ is trivial, $T \mathbb{R}^{3} \equiv \mathbb{R}^{3} \times \mathbb{R}^{3}$, for each $j \in E\left(M ; \mathbb{R}^{3}\right)$ the tangent map $T j: T M \rightarrow T \mathbb{R}^{3}$ splits $T j=(j, d j)$. In classical terms an embedding $j$ is called a placement of the body $M$ and the exact $\mathbb{R}^{3}$-valued one-form $d j \in \mathcal{I}\left(M ; \mathbb{R}^{3}\right)$ corresponds to the deformation gradient.

Following [28], we consider a material whose interior structure is given by some triple of vector fields $X_{1}, X_{2}, X_{3} \in \Gamma(T M)$ such that

$$
\gamma_{\mathrm{ex}}\left(X_{l}\right)=e_{l}, \quad l=1,2,3
$$

where $\gamma_{\mathrm{ex}}=d j_{0}$ for some reference embedding $j_{0} \in E\left(M ; \mathbb{R}^{3}\right)$. Since $d^{2}=0$, it follows from that discussion above that this triple $X_{1}, X_{2}, X_{3} \in \Gamma(T M)$ characterizes a nondislocated material. Let $j \in E\left(M ; \mathbb{R}^{3}\right)$ be an arbitrary but fixed embedding and $\beta \in \mathcal{D}\left(M ; \mathbb{R}^{3}\right)$ an arbitrary but fixed divergence-free one-form which, according to Sec. 3 , describes the presence of dislocations. Consider now the form $(d j+\beta) \in \Omega^{1}\left(M ; \mathbb{R}^{3}\right)$. Then the frame $X_{1}, X_{2}, X_{3}$ induces a triple of vector fields on $j(M) \subset \mathbb{R}^{3}$, given by

$$
(d j+\beta)\left(X_{1}\right), \quad(d j+\beta)\left(X_{2}\right), \quad(d j+\beta)\left(X_{3}\right) .
$$

For $\beta$ sufficiently small, ${ }^{1}$ this triple constitutes a parallelization and hence a global frame on $j(M) \subset \mathbb{R}^{3}$, if and only if $(d j+\beta) \in \mathcal{I}\left(M ; \mathbb{R}^{3}\right)$. In particular, this frame represents a dislocated lattice on the embedded body $j(M)$. In other words, the original frame $d j_{0} X_{1}, d j_{0} X_{2}, d j_{0} X_{3}$ on $j_{0}(M)$ is deformed incompatibly with respect to the original nondislocated reference lattice. Since $d^{2}=0, d(d j+\beta)=d \beta$, which in turn implies that dislocations are encoded in the nonexact component $\beta \in \mathcal{D}\left(M ; \mathbb{R}^{3}\right)$ of $(d j+\beta)$.

The observation is that up to rigid translations, only the integrable component of the generalized configuration $\gamma=d j+\beta$ determines the embedding $j$ of the body manifold in the physical space $\mathbb{R}^{3}$. On the contrary, the divergence-free component $\beta \in \mathcal{D}\left(M ; \mathbb{R}^{3}\right)$ describes the discrepancy between the macroscopic placement and the lattice. Thus, as an appropriate framework for describing elastic materials with dislocations, we consider so-called generalized configurations $\gamma=d j+\beta \in \mathcal{I}\left(M ; \mathbb{R}^{3}\right)$ whose integrable component

\footnotetext{
${ }^{1}$ This can be made precise in mathematical terms, cf. [28].
} 
$d j$ stems from a placement $j \in E\left(M ; \mathbb{R}^{3}\right)$ and whose nonintegrable component $\beta$ lies in $\mathcal{D}\left(M ; \mathbb{R}^{3}\right)$. The set of all such configurations is denoted by

$$
\mathcal{V}\left(M ; \mathbb{R}^{3}\right)=\left\{d j+\beta \in \mathcal{I}\left(M ; \mathbb{R}^{3}\right) \mid j \in E\left(M ; \mathbb{R}^{3}\right), \beta \in \mathcal{D}\left(M ; \mathbb{R}^{3}\right)\right\} .
$$

Observe that $\mathcal{V}\left(M ; \mathbb{R}^{3}\right)$ contains the space of all deformation gradients

$$
d E\left(M ; \mathbb{R}^{3}\right):=\left\{d j \in \Omega^{1}\left(M ; \mathbb{R}^{3}\right) \mid j \in E\left(M ; \mathbb{R}^{3}\right)\right\}
$$

as an open subset. In classical elasticity, the crucial kinematic quantity is the deformation gradient $d j$ of an embedding $j$. Therefore, the classical configuration space of elasticity $E\left(M ; \mathbb{R}^{3}\right)$ is replaced here by $d E\left(M ; \mathbb{R}^{3}\right)$, cf. [16]. Since $d E\left(M ; \mathbb{R}^{3}\right)$ is a Fréchet submanifold of $\mathcal{V}\left(M ; \mathbb{R}^{3}\right)$, the Fréchet manifold $\mathcal{V}\left(M ; \mathbb{R}^{3}\right)$ is a natural generalization of the configuration space $d E\left(M ; \mathbb{R}^{3}\right)$ of defect-free continuum mechanics. In fact, one can show that any possible dislocation density can be described in terms of generalized configurations $\gamma \in \mathcal{V}\left(M ; \mathbb{R}^{3}\right)$, cf. [27].

5. The geometry of $\mathcal{V}\left(M ; \mathbb{R}^{3}\right)$. For a mathematical formulation of a dynamic theory for dislocated materials, we need a metric on the configuration space $\mathcal{V}\left(M ; \mathbb{R}^{3}\right)$. The most natural choice for such a metric would be the inner product $\mathcal{G}$ given in $(2)$ restricted to our configuration space $\mathcal{V}\left(M ; \mathbb{R}^{3}\right)$. This metric, however, has to be rejected for physical reasons, since it does not cover the case of a defect-free elastic solid. Instead, we first introduce an adapted metric on $d E\left(M ; \mathbb{R}^{3}\right)$, following the approach in [6]. Let $\rho: M \rightarrow \mathbb{R}$ be a positive function and define

$$
C: C^{\infty}\left(M ; \mathbb{R}^{3}\right) \rightarrow \mathbb{R}^{3}, \quad C[u]:=\int_{M} \rho u \mu, \quad u \in C^{\infty}\left(M ; \mathbb{R}^{3}\right) .
$$

Then each $u \in C^{\infty}\left(M ; \mathbb{R}^{3}\right)$ uniquely splits into

$$
u=u^{0}+C[u], \quad \text { where } \int_{M} \rho u^{0} \mu=0 .
$$

Since $E\left(M ; \mathbb{R}^{3}\right) \subset C^{\infty}\left(M ; \mathbb{R}^{3}\right)$ is open, we can decompose each $j \in E\left(M ; \mathbb{R}^{3}\right)$ accordingly into

$$
j=j^{0}+C[j], \quad \text { where } \int_{M I} \rho j^{0} \mu=0 .
$$

Physically, the vector $C[j] \in \mathbb{R}^{3}$ points towards the center of mass of the embedded body $j(M)$ in $\mathbb{R}^{3}$. Let

$$
E_{0}\left(M ; \mathbb{R}^{3}\right):=\left\{j \in E\left(M ; \mathbb{R}^{3}\right) \mid \int_{M} \rho j \mu=0\right\}
$$

denote the space of all configurations in $E\left(M ; \mathbb{R}^{3}\right)$ that keep the center of mass fixed: $C[j]=0$. The space $E_{0}\left(M ; \mathbb{R}^{3}\right)$ is a Fréchet manifold that is naturally isomorphic to $d E\left(M ; \mathbb{R}^{3}\right)$, cf. $[3,4]$. Since $d E\left(M ; \mathbb{R}^{3}\right) \subset d C^{\infty}\left(M ; \mathbb{R}^{3}\right)$ is open,

$$
T\left(d E\left(M ; \mathbb{R}^{3}\right)\right)=d E\left(M ; \mathbb{R}^{3}\right) \times d C^{\infty}\left(M ; \mathbb{R}^{3}\right) .
$$

A metric on $d E\left(M ; \mathbb{R}^{3}\right)$ naturally induced by this construction is given by

$$
\mathcal{G}_{E}\left(d u_{1}, d u_{2}\right):=\int_{M} \rho\left\langle u_{1}^{0}, u_{2}^{0}\right\rangle_{\mathbb{R}^{3}} \mu, \quad d u_{1}, d u_{2} \in d C^{\infty}\left(M ; \mathbb{R}^{3}\right) .
$$


Here we identify tangent vectors with their principal parts.

Since the configuration space $\mathcal{V}\left(M ; \mathbb{R}^{3}\right)$ is an open subset of $\Omega^{1}\left(M ; \mathbb{R}^{3}\right)$, the tangent manifold $T \mathcal{V}\left(M ; \mathbb{R}^{3}\right)$ of $\mathcal{V}\left(M ; \mathbb{R}^{3}\right)$ is trivial, that is,

$$
T \mathcal{V}\left(M ; \mathbb{R}^{3}\right)=\mathcal{V}\left(M ; \mathbb{R}^{3}\right) \times \Omega^{1}\left(\Lambda I \mathbb{R}^{3}\right) .
$$

An application of Theorem 2.1 to tangent vectors $\xi \in T \mathcal{V}\left(M ; \mathbb{R}^{3}\right)$ allows us to equip the configuration space $\mathcal{V}\left(M ; \mathbb{R}^{3}\right)$ with a metric as follows.

Definition 5.1. Let $\gamma \in \mathcal{V}\left(M ; \mathbb{R}^{3}\right)$ be an arbitrary generalized configuration. For each pair $\xi_{i} \in T_{\gamma} \mathcal{V}\left(M ; \mathbb{R}^{3}\right), i=1,2$, let

$$
\xi_{i}=d u_{i}+\chi_{i} \quad \text { with } d u_{i} \in d C^{\infty}\left(M ; \mathbb{R}^{3}\right), \chi_{i} \in \mathcal{D}\left(M ; \mathbb{R}^{3}\right)
$$

be the respective Helmholtz decompositions. A metric $\mathcal{G}_{\mathcal{V}}$ on the configuration space $\mathcal{V}\left(M ; \mathbb{R}^{3}\right)$ is defined by setting

$$
\mathcal{G}_{\mathcal{V}}[\gamma]\left(\xi_{1}, \xi_{2}\right):=\mathcal{G}_{\mathcal{V}}^{(e)}[\gamma]\left(d u_{1}, d u_{2}\right)+\mathcal{G}_{\mathcal{V}}^{(p)}[\gamma]\left(\chi_{1}, \chi_{2}\right)
$$

The elastic component of $\mathcal{G}_{\mathcal{V}}$ is given by

$$
\mathcal{G}_{\mathcal{V}}^{(e)}[\gamma]\left(d u_{1}, d u_{2}\right):=\mathcal{G}_{E}\left(d u_{1}, d u_{2}\right), \quad d u_{1}, d u_{2} \in d C^{\infty}\left(M ; \mathbb{R}^{3}\right),
$$

where $\mathcal{G}_{E}$ is defined in (17). The plastic component of $\mathcal{G}_{\mathcal{V}}$ is given by

$$
\mathcal{G}_{\mathcal{V}}^{(p)}[\gamma]\left(\chi_{1}, \chi_{2}\right):=\int_{M} \sigma\left\langle\chi_{1}, \chi_{2}\right\rangle \mu, \quad \chi_{1}, \chi_{2} \in \mathcal{D}\left(M ; \mathbb{R}^{3}\right),
$$

where $\sigma \in C^{\infty}(M I)$ is a strictly positive real-valued function.

Notice that the densities $\rho$ and $\sigma$ appearing in $\mathcal{G}_{\mathcal{V}}^{(e)}$ and $\mathcal{G}_{\mathcal{V}}^{(p)}$, respectively, are defined on the body manifold $M$. In this sense $\sigma$ appearing in the metric $\mathcal{G}_{\mathcal{V}}^{(p)}$ may be thought of as the density of inertia of the dislocations. For sake of simplicity, we assume that the density $\sigma$ is independent of the actual configuration $\gamma$. In the physical space $\mathbb{R}^{3}$, however, both densities appear as push-forwards $j_{*} \rho:=\rho \circ j^{-1}$ and $j_{*} \sigma:=\sigma \circ j^{-1}$ of the actual configuration $j$. The choice of the metric gives rise to define the kinetic energy functional $\mathcal{E}: T \mathcal{V}\left(M ; \mathbb{R}^{3}\right) \rightarrow \mathbb{R}$ by setting

$$
\mathcal{E}(\xi):=\frac{1}{2} \mathcal{G}_{\mathcal{V}}[\gamma](\xi, \xi), \quad \xi \in T_{\gamma} \mathcal{V}\left(m ; \mathbb{R}^{3}\right), \quad \gamma \in \mathcal{V}\left(\Lambda ; \mathbb{R}^{3}\right) .
$$

If $\xi=d u+\chi$ denotes the Helmholtz decomposition, the kinetic energy $\mathcal{E}$ splits into an elastic component and a plastic component, given by

$$
\mathcal{E}^{(e)}(\xi):=\frac{1}{2} \mathcal{G}_{\mathcal{V}}^{(e)}[\gamma](d u, d u) \text { and } \mathcal{E}^{(p)}(\xi):=\frac{1}{2} \mathcal{G}_{\mathcal{V}}^{(p)}[\gamma](\chi, \chi)
$$

respectively. The functional $\mathcal{E}^{(e)}(\cdot): T \mathcal{V}\left(M ; \mathbb{R}^{3}\right) \rightarrow \mathbb{R}$ corresponds to the kinetic energy associated with purely elastic deformations, whereas the functional $\mathcal{E}^{(p)}(\cdot): T \mathcal{V}\left(M ; \mathbb{R}^{3}\right) \rightarrow$ $\mathbb{R}$ corresponds to the kinetic energy associated with the dislocation density. By construction, the metric $\mathcal{G}_{\mathcal{V}}$ is constant in $\gamma$, that is,

$$
D \mathcal{G}_{\mathcal{V}}[\gamma](\eta)=0 \quad \forall \eta \in T_{\gamma} \mathcal{V}\left(M ; \mathbb{R}^{3}\right), \quad \gamma \in \mathcal{V}\left(M ; \mathbb{R}^{3}\right)
$$

Therefore, the corresponding Euler's equations yield

$$
\mathcal{G}_{\mathcal{V}}[\gamma(t)](\ddot{\gamma}(t), \eta)=0 \quad \forall \eta \in T \mathcal{V}\left(M ; \mathbb{R}^{3}\right)
$$

as weak equations of motion in the absence of internal interactions of the material. Analogously to elasticity, the solution to the geodesic equation $(19)$ is $\dot{\gamma}=0$, cf. $[4,6]$. 
A motion of a material with nonvanishing internal interactions will, of course, deviate from these geodesics. The resulting dynamics will be discussed in Sec. 6 .

To put Euler's equations into a more geometric framework, we introduce a symplectic structure on $T \mathcal{V}\left(M ; \mathbb{R}^{3}\right)$. Closing this section, we show that the symplectic set-up here becomes trivial. Let $T \pi_{\mathcal{V}}: T^{2} \mathcal{V}\left(M ; \mathbb{R}^{3}\right) \rightarrow T \mathcal{V}\left(M ; \mathbb{R}^{3}\right)$ denote the tangent map of the canonical projection $\pi_{\mathcal{V}}$ and $V\left(T \mathcal{V}\left(M ; \mathbb{R}^{3}\right)\right):=\operatorname{ker} T \pi_{\mathcal{V}}$ the vertical bundle. If $V \mathcal{X} \in$ $V\left(T \mathcal{V}\left(M ; \mathbb{R}^{3}\right)\right)$ denotes the vertical component of any vector $\mathcal{X} \in T^{2} \mathcal{V}\left(M ; \mathbb{R}^{3}\right)$, the metric $\mathcal{G}_{\mathcal{V}}$ defines a natural weakly nondegenerate symplectic two-form $\Omega$ on $T \mathcal{V}\left(M ; \mathbb{R}^{3}\right)$, given by

$$
\Omega[\xi](\mathcal{X}, \mathcal{Y}):=\mathcal{G}_{\mathcal{V}}[\gamma]\left(V \mathcal{Y}, T \pi_{\mathcal{V}} \mathcal{X}\right)-\mathcal{G}_{\mathcal{V}}[\gamma]\left(V \mathcal{X}, T \pi_{\mathcal{V}} \mathcal{Y}\right)
$$

for all $\mathcal{X}, \mathcal{Y} \in T_{\xi} T \mathcal{V}\left(M ; \mathbb{R}^{3}\right), \xi \in T_{\gamma} \mathcal{V}\left(M ; \mathbb{R}^{3}\right), \gamma \in \mathcal{V}\left(M ; \mathbb{R}^{3}\right)$. Thus, $T \mathcal{V}\left(M ; \mathbb{R}^{3}\right)$ endowed with $\Omega$ becomes a symplectic manifold. Since $T \mathcal{V}\left(M ; \mathbb{R}^{3}\right)$ is trivial, in coordinates one has

$$
\mathcal{X}=\left(\gamma, \xi, \zeta_{1}, \zeta_{2}\right) \text { and } \mathcal{Y}=\left(\gamma, \xi, \eta_{1}, \eta_{2}\right)
$$

which in turn yields

$$
\Omega[\gamma, \xi]\left(\left(\zeta_{1}, \zeta_{2}\right),\left(\eta_{1}, \eta_{2}\right)\right)=\mathcal{G}_{\mathcal{V}}[\gamma]\left(\eta_{2}, \zeta_{1}\right)-\mathcal{G}_{\mathcal{V}}[\gamma]\left(\zeta_{2}, \eta_{1}\right)
$$

6. The principle of virtual work. A work functional on the space of generalized configurations $\mathcal{V}\left(M ; \mathbb{R}^{3}\right)$ is understood to be a continuous linear functional

$$
F: T \mathcal{V}\left(M ; \mathbb{R}^{3}\right) \equiv \mathcal{V}\left(M ; \mathbb{R}^{3}\right) \times \Omega^{1}\left(M ; \mathbb{R}^{3}\right) \rightarrow \mathbb{R}
$$

on the tangent bundle $T \mathcal{V}\left(M ; \mathbb{R}^{3}\right)$. We assume that for each configuration $\gamma \in \mathcal{V}\left(M ; \mathbb{R}^{3}\right)$ the functional $F$ admits an integral representation with respect to the metric $\mathcal{G}$ given in (2) such that

$$
F[\gamma](\eta)=\int_{M}\langle\alpha[\gamma], \eta\rangle \mu \quad \forall \eta \in T_{\gamma} \mathcal{V}\left(M ; \mathbb{R}^{3}\right)
$$

The constitutive law ${ }^{2}$ of the material $M$ is encoded in the functional dependence of the integral kernel $\alpha[\gamma] \in \Omega^{1}\left(M ; \mathbb{R}^{3}\right)$ on the configuration $\gamma$. This dependence will, in general, be nonlinear and possibly also nonlocal. More precisely, the integral kernel $\alpha$ may be thought of as a smooth section into the tangent bundle $T \mathcal{V}\left(M ; \mathbb{R}^{3}\right)$, where each $\alpha[\gamma]$ is identified with its principal part. The one-form $\alpha$ is called a (generalized) stress form. In classical elasticity, $\alpha$ corresponds to the first Piola-Kirchhoff stress tensor, cf. $[16,21]$.

For each $\gamma \in \mathcal{V}\left(M ; \mathbb{R}^{3}\right)$, the Helmholtz decomposition of $\alpha[\gamma]$ reads

$$
\alpha[\gamma]=d h[\gamma]+\tau[\gamma]
$$

\footnotetext{
${ }^{2}$ If the system is integrable, then there exists a potential energy functional $U: \mathcal{V}\left(M ; \mathbb{R}^{3}\right) \rightarrow \mathbb{R}^{3}$ such that $F[\gamma](\eta)=D U[\gamma](\eta)$, with $D$ denoting the Fréchet derivative on the space of functions on $\mathcal{V}\left(M ; \mathbb{R}^{3}\right)$. However, such an "integral" a priori need not exist and, in fact, will not exist in the presence of dissipative effects, cf. [7]. For this reason we consider work functionals instead of potential energy functionals.
} 
where $d h[\gamma] \in d C^{\infty}\left(M ; \mathbb{R}^{3}\right)$ is a gradient and $\tau[\gamma] \in \mathcal{D}\left(M ; \mathbb{R}^{3}\right)$ is divergence-free. Writing $\eta=d u+\chi$, the orthogonality of the Helmholtz decomposition, Theorem 2.1, implies

$$
\mathcal{G}(\alpha[\gamma], \eta)=\mathcal{G}(d h[\gamma], d u)+\mathcal{G}(\tau[\gamma], \chi) .
$$

Therefore, for each generalized configuration $\gamma \in \mathcal{V}\left(M ; \mathbb{R}^{3}\right)$, the work functional $F$ splits into an elastic component $F^{(e)}$ and a plastic component $F^{(p)}$, i.e.,

$$
F[\gamma](\eta)=F^{(e)}[\gamma](d u)+F^{(p)}[\gamma](\chi) \quad \forall \eta=d u+\chi \in T_{\gamma} \mathcal{V}\left(M ; \mathbb{R}^{3}\right) .
$$

The elastic component is given by

$$
F^{(e)}[\gamma](d u):=\int_{M}\langle d h[\gamma], d u\rangle_{\mu} \quad \forall d u \in d C^{\infty}\left(M ; \mathbb{R}^{3}\right)
$$

and the plastic component by

$$
F^{(p)}[\gamma](\chi):=\int_{M}\langle\tau[\gamma], \chi\rangle \mu \quad \forall \chi \in \mathcal{D}\left(M ; \mathbb{R}^{3}\right) .
$$

Since the Helmholtz decomposition is orthogonal,

$$
F=F^{(e)} \Leftrightarrow \alpha[\gamma]=d h[\gamma] \quad \forall \gamma \in \mathcal{V}\left(M ; \mathbb{R}^{3}\right) .
$$

The second Piola-Kirchhoff stress tensor $\boldsymbol{S}[\gamma]$ associated with the stress form $\alpha[\gamma] \in$ $\Omega^{1}\left(M ; \mathbb{R}^{3}\right), \gamma \in \mathcal{V}\left(M ; \mathbb{R}^{3}\right)$ is given by

$$
\boldsymbol{S}[\gamma](X, Y):=\langle\alpha[\gamma](X), \gamma(Y)\rangle_{\mathbb{R}^{3}}, \quad X, Y \in \Gamma(T M) .
$$

Observe that the stress form associated with the Piola-Kirchhoff stress tensor $\boldsymbol{S}[\gamma]$ will, in general, be nonexact, that is, $\alpha[\gamma] \neq d h[\gamma]$. However, only the exact component $d h[\gamma]$ of the stress form $\alpha[\gamma]$ contributes to the work functional $F^{(e)}$, cf. [3, 5]. In fact, $F^{(e)}$ is the well-known work functional in elasticity, cf. $[1,7,18]$. The work functional $(22)$ thus becomes a natural generalization of the notion of work in elasticity.

Since in pure elasticity only the integrable component $d h[\gamma]$ of a stress form $\alpha[\gamma]$ contributes to the work functional of elasticity $F^{(e)}$, any stress form $\tilde{\alpha}[\gamma]=\alpha[\gamma]+\chi[\gamma]$ with arbitrary $\chi[\gamma] \in \mathcal{D}\left(M ; \mathbb{R}^{3}\right)$ will represent the same work functional $F^{(e)}$, cf. [3]. In particular, one may choose $\chi[\gamma]$ such that the stress tensor $\tilde{\boldsymbol{S}}$ corresponding to $\tilde{\alpha}[\gamma]$ is symmetric, cf. [21].

In the dislocated case, this gauge freedom is lost. Since the divergence-free component $\tau[\gamma]$ of the stress form $\alpha$ appears explicitly in the principle of virtual work (29), the stress tensor cannot be symmetric. Both components $d h[\gamma]$ and $\tau[\gamma]$ of the stress form $\alpha[\gamma]=d h[\gamma]+\tau[\gamma]$ will, in general, depend on the integrable component $d j$ as well as the plastic component $\beta \in \mathcal{D}\left(M ; \mathbb{R}^{3}\right)$ of $\gamma=d j+\beta$. Hence, $\tau[\gamma]$ describes the plastic components acting on the dislocations of the material in view. According to the Hodge decomposition, each $\tau \in \mathcal{D}\left(M ; \mathbb{R}^{3}\right)$ may uniquely be decomposed into $\tau=\delta \zeta+\kappa$, with a two-form $\zeta \in \Omega^{2}\left(M ; \mathbb{R}^{3}\right)$ and a so-called harmonic component $\kappa$. The latter depends exclusively on the topology of $M$, and vanishes if the body manifold $M$ is simply connected. In this case $\tau=\delta \zeta$ and in the sense of [13], $\zeta$ may be interpreted as a moment stress (form).$^{3}$

\footnotetext{
${ }^{3}$ The concept of decomposing configurations $\tau \in \mathcal{V}\left(M ; \mathbb{R}^{3}\right)$ and stress forms $\alpha[\gamma] \in \Omega^{1}\left(M ; \mathbb{R}^{3}\right)$, $\gamma \in \mathcal{V}\left(M ; \mathbb{R}^{3}\right)$ is completely analogous to the concept of strain spaces and stress spaces in [12]. The integrable component of the deformation is the dual quantity to the integrable component of the stress,
} 
Next, we implement the work functional (22) in the d'Alembert principle of virtual work. According to [17], an exterior force acting on a general mechanical system is given by a horizontal one-form on the tangent manifold of the corresponding configuration space. A vector field $\mathcal{Y}$ on $T \mathcal{V}\left(M ; \mathbb{R}^{3}\right)$ is by definition vertical, if $T \pi_{\mathcal{V}}(\mathcal{Y})=0$, where $T \pi_{\mathcal{V}}$ denotes the tangent map of the canonical projection $\pi_{\mathcal{V}}$. A one-form $\mathcal{F}$ on $T \mathcal{V}\left(M ; \mathbb{R}^{3}\right)$ is horizontal, if $\mathcal{F}(\mathcal{Y})=0$ for all vertical vector fields $\mathcal{Y}$. Thus, an exterior force in the above sense acting on dislocated material is given by a horizontal one-form $\mathcal{F}$ on $T \mathcal{V}\left(M ; \mathbb{R}^{3}\right)$. Using $T \pi_{\mathcal{V}}$, the work functional $F$ defined in (22) induces an exterior work one-form $\mathcal{F}$ in the above sense by setting

$$
\mathcal{F}:=\left(T \pi_{\mathcal{V}}\right)^{*} F
$$

Due to the pull-back construction, $\mathcal{F}$ is horizontal.

On the other hand, if $\mathcal{Y}$ is a vertical vector field and $\Omega$ is the symplectic two-form defined in (20), then

$$
\Omega(\mathcal{Y}, \mathcal{Z})=-\mathcal{G}_{\mathcal{V}}[\gamma]\left(\mathcal{Y}, T \pi_{\mathcal{V}} \mathcal{Z}\right) \quad \forall \mathcal{Z} \in \Gamma\left(T^{2} \mathcal{V}\left(M ; \mathbb{R}^{3}\right)\right)
$$

Therefore, the induced one-form $\iota y \Omega$ given by

$$
\iota \mathcal{Y} \Omega(\mathcal{Z}):=\Omega(\mathcal{Y}, \mathcal{Z}) \quad \forall \mathcal{Z} \in \Gamma\left(T^{2} \mathcal{V}\left(M ; \mathbb{R}^{3}\right)\right)
$$

is horizontal. Given the kinetic energy functional $\mathcal{E}$ and an exterior work one-form (27), the d'Alembert principle of virtual work now states that the Euler vector field $\mathcal{X}$ is determined by the equation

$$
d \mathcal{E}(\mathcal{Z})-\iota_{\mathcal{X}} \Omega(\mathcal{Z})=\left(T \pi_{\mathcal{V}}\right)^{*} F(\mathcal{Z}) \quad \forall \mathcal{Z} \in \Gamma\left(T^{2} \mathcal{V}\left(M ; \mathbb{R}^{3}\right)\right)
$$

7. The equations of motion. In order to formulate a dynamics on our configuration space $\mathcal{V}\left(M ; \mathbb{R}^{3}\right)$, consider a motion given by a smooth curve

$$
\gamma:[0, T] \rightarrow \mathcal{V}\left(M ; \mathbb{R}^{3}\right), \quad t \mapsto \gamma(t) .
$$

Using the exterior work functional (27), the curve $\gamma(t)$ describes a motion subject to the d'Alembert principle of virtual work (28), if it satisfies the weak equations of motion

$$
\mathcal{G}_{\mathcal{V}}[\gamma(t)]\left(\partial_{t}^{2} \gamma(t), \eta\right)=F[\gamma(t)](\eta) \quad \forall \eta \in \Omega^{1}\left(M ; \mathbb{R}^{3}\right) .
$$

According to Helmholtz, each $\gamma(t), t \in[0, T]$ decomposes into

$$
\gamma(t)=d j_{\gamma}(t)+\beta_{\gamma}(t)
$$

The orthogonality of the splittings of the work functional $F=F^{(e)}+F^{(p)}$ and the metric $\mathcal{G}_{\mathcal{V}}=\mathcal{G}_{\mathcal{V}}^{(e)}+\mathcal{G}_{\mathcal{V}}^{(p)}$ given in Definition 5.1 implies that $(29)$ is equivalent to the system of equations

$$
\mathcal{G}_{\mathcal{V}}^{(e)}[\gamma(t)]\left(\partial_{t}^{2} d j_{\gamma}(t), d u\right)=F^{(e)}[\gamma(t)](d u) \quad \forall d u \in d C^{\infty}\left(M ; \mathbb{R}^{3}\right)
$$

and

$$
\mathcal{G}_{\mathcal{V}}^{(p)}[\gamma(t)]\left(\partial_{t}^{2} \beta_{\gamma}(t), \chi\right)=F^{(p)}[\gamma(t)](\chi) \quad \forall \chi \in \mathcal{D}\left(M ; \mathbb{R}^{3}\right) .
$$

the nonintegrable component of the deformation is the dual quantity to the nonintegrable component of the stress. This nonintegrable component plays the role of the moment stress tensor. 
Thus, the dynamical equations derived from the principle of virtual work split into an elastic component (30) and into a plastic component (31). In the absence of external volume forces, the equations of motion ${ }^{4}$ induced by (30) and (31) are given by an application of Stokes' Theorem for differential forms (see [16]).

TheOREM 7.1. Let $\alpha[\gamma]=d h[\gamma]+\tau[\gamma]$ be the Helmholtz decomposition of the stress form for a dislocated material. Then the equations of motion are given by

$$
\begin{aligned}
\rho \partial_{t}^{2} j_{\gamma}(t) & =\Delta h[\gamma(t)], \\
\sigma \partial_{t}^{2} \beta_{\gamma}(t) & =\tau[\gamma(t)] .
\end{aligned}
$$

Here $\gamma(t)=d j_{\gamma}(t)+\beta_{\gamma}(t)$ is the Helmholtz decomposition of the actual configuration $\gamma(t)$ and $\Delta:=\delta \circ d$ is the Laplace operator on functions in $C^{\infty}\left(M ; \mathbb{R}^{3}\right)$.

Since $j_{\gamma}$ and $\beta_{\gamma}$ both have values in $\mathbb{R}^{3}$, the equations of motion (32) are 6 coupled scalar equations for 6 unknown fields which form a system of nonlinear partial differential equations coupled via the Helmholtz decomposition. ${ }^{5}$ In this full generality, it is beyond the scope of this paper to say something about the well-posedness of this problem. However, the first equation in Theorem 7.1 is the equation of motion obtained in $[9$, p. 99] (see also $[15$, p. 137]): since $\delta \tau[\gamma]=0$, the divergence of the stress form $\alpha[\gamma]$ corresponding to the first Piola-Kirchhoff stress tensor can be represented as the Laplace operator on functions, that is, $\delta \alpha[\gamma]=\Delta h[\gamma]$. The second one is an evolution equation for the nonintegrable components $\beta_{\gamma}(t)$ and, hence, of the dislocation densities associated with the deformation $\gamma(t)$. The time derivatives $\dot{\beta}_{\gamma}(t)$ correspond to what is called the dislocation flux density tensor (cf. [15, p. 137]) and

$$
\dot{b}(t)=\int_{S} d \dot{\beta}_{\gamma}(t)
$$

gives the flux of the Burgers vector through the surface $S$. Observe that our configuration space $\mathcal{V}\left(M ; \mathbb{R}^{3}\right)$ is designed in such a way that the so-called conservation laws in [9] are satisfied by construction.

In physics, the constitutive behavior of the material such as Hooke's law is usually given as the functional dependence of the stress on the strain. In our approach this corresponds to a functional relationship between the stress form $\alpha[\gamma]$ and the configuration $\gamma$. In order to obtain the coupled system of (32), the nonexact component $\tau[\gamma]$ from a given stress form $\alpha[\gamma]$ and hence its Helmholtz decomposition has to be computed. The problem of determining the nonexact component $\tau[\gamma]$, or, equivalently, its exact component $d h[\gamma]$ amounts to solving a boundary value problem of the Neumann type.

Proposition 7.1. Let $M$ be a simply connected 3-dimensional manifold with boundary $\partial M$ and $\mathcal{N}$ be the unit normal field. Assume that the stress form $\alpha[\gamma]$ is given. Then

\footnotetext{
${ }^{4}$ The equivalence of the weak equations and the strong equations follows from the fact that the space of smooth differential forms is dense in an appropriate $L^{2}$-completion, cf. [22].

${ }^{5}$ Observe that we assume that this decomposition is with respect to a fixed reference metric. However, this reference metric could, in principle, change with the configuration.
} 
the boundary value problem

$$
\begin{aligned}
\delta d h[\gamma] & =\delta \alpha[\gamma] & & \text { on } M \\
d h[\gamma](\mathcal{N}) & =\alpha[\gamma](\mathcal{N}) & & \text { on } \partial M
\end{aligned}
$$

has a unique solution.

For a proof see [22, Corollary 3.4 .8$, p. 135]. In the case where $M$ is not simply connected, a solution to the Neumann problem still exists but is not unique. Since $M \subset \mathbb{R}^{3}$, there exists an explicit integral representation for the solution $d h[\gamma]$, cf. [10]. This determines $\tau[\gamma]=\alpha[\gamma]-d h[\gamma]$ explicitly. Also, as mentioned above, in the simply connected case there exists a moment stress from $\zeta[\gamma]$ with $\tau[\gamma]=\delta \zeta[\gamma]$. The static case of Theorem 7.1 gives an analogue to equations obtained in $[11$, p. 306]. Formally we state:

Corollary 7.1. A configuration $\gamma_{0} \in \mathcal{V}\left(M ; \mathbb{R}^{3}\right)$ is an equilibrium configuration if and only if

$$
\Delta h\left[\gamma_{0}\right]=0 \quad \text { and } \quad \tau\left[\gamma_{0}\right]=0
$$

The motion of dislocations will, in general, be accompanied by dissipative effects and irreversibility, cf. [13]. The equations in Theorem 7.1 contain only second derivatives with respect to time and therefore cannot account for these effects. To complete our dynamical description, one would have to incorporate the thermodynamics of dislocations, which is beyond the scope of this paper. We refer the interested reader to [19] and finish instead with a simple example indicating how irreversible effects could be incorporated into our framework.

EXAMPLE. Let the virtual work functional $F$ admit an integral representation of the form (22) with a stress form

$$
\alpha[\gamma]=A \circ d j_{\gamma}+f\left(\left\|d j_{\gamma}\right\|^{2}\right) \beta_{\gamma},
$$

where $A$ is a smooth bundle endomorphism (i.e., each $A_{p}: \mathbb{R}^{3} \rightarrow \mathbb{R}^{3}, p \in M$ is linear with smooth dependence on $p \in M)$ and $\left\|d j_{\gamma}\right\|^{2}:=\int_{M}\left\langle d j_{\gamma}, d j_{\gamma}\right\rangle \mu$. As before, the configuration $\gamma$ is decomposed according to Helmholtz into $\gamma=d j_{\gamma}+\beta_{\gamma}$ and $f: \mathbb{R} \rightarrow \mathbb{R}$ is a smooth function defined by $f(s)=0$ for $s \leq a_{\text {crit }}$ and $f(s)=\exp \left(-1 /\left(s-a_{\text {crit }}\right)\right)$ for $s>a_{\text {crit }}$.

The bundle endomorphism $A$ may play the role of Hooke's law (e.g., see [28]) and $\left\|d j_{\gamma}\right\|^{2}$ is a measure for the volume of the configuration $\gamma$. Assuming that $A \circ d j_{\gamma}$ is an exact one-form, the equations of motion (32) given in Theorem 7.1 reduce to

$$
\rho \partial_{t}^{2} j_{\gamma}=\delta\left(A \circ d j_{\gamma}\right)
$$

for the elastic components and

$$
\sigma \partial_{t}^{2} \beta_{\gamma}=f\left(\left\|d j_{\gamma}\right\|^{2}\right) \beta_{\gamma}
$$

for the plastic components. This implies that for small deformations, i.e., as long as $\left\|d j_{\gamma}\right\|^{2}<a_{\text {crit }}$, an initial defect structure will only be deformed elastically. However, as soon as $\left\|d j_{\gamma}\right\|^{2}$ exceeds the critical value $a_{\text {crit }}$, the plastic components tend to grow exponentially. 
Although this example may be too simple to describe real materials, it shows that irreversible effects may be included into our setup. In any case, a proper choice of the constitutive law for the stress form $\alpha[\gamma]$ has to be made on the basis of phenomenological reasoning from physical experience. This, however, is again beyond the scope of this paper.

\section{REFERENCES}

[1] S. S. Antman and J. E. Osborne, The principle of virtual work and integral laws of motion, Arch. Rational Mech. Anal. 69, 231-262 (1979)

[2] E. Binz, J. Śniatycki, and H. Fischer, Geometry of Classical Fields, Mathematics Studies 154, North-Holland, 1988

[3] E. Binz, Symmetry, constitutive laws of bounded smoothly deformable media and Neumann problems, In: Symmetry in Science V, ed. B. Gruber, L. C. Biedenharn, and H. D. Doebner, Plenum Press, New York, London, 1991

[4] E. Binz, Global differential geometric methods in elasticity and hydrodynamics, In: Differential Geometry, Group Representations and Quantization, ed. J. B. Hennig, W. Lücke, and J. Tolar, Lecture Notes in Physics 379, 3-28 (1991)

[5] E. Binz, On the irredundant part of the first Piola-Kirchhoff stress tensor, Rep. Math. Phys. 32, 175-210 (1993)

[6] E. Binz and G. Schwarz, The principle of work and a symplectic reduction of nonlocal continuum mechanics, Rep. Math. Phys. 32, 49-69 (1993)

[7] M. Epstein and R. Segev, Differential manifolds and the principle of virtual work in continuum mechanics, J. Math. Phys. 21, 1243-1245 (1980)

[8] E. Hellinger, Die allgemeinen Ansätze der Mechanik der Kontinua, Enzykl. Math. Wiss. 4/4 (1914)

[9] A. M. Kosevich, Crystal dislocations and the theory of elasticity, In: Dislocations in Solids, ed. F. R. N. Nabarro, North Holland, Amsterdam, 1979, pp. 33-165

[10] R. Kress, Potentialtheoretische Randwertproblem bei Tensorfeldern beliebiger Dimension und beliebigen Ranges, Arch. Rational Mech. Anal. 47, 59-80 (1972)

[11] E. Kröner, Continuum Theory of Defects, Les Houches, Session XXXV, 1980-Physics of Defects, R. Balian et al., eds., North-Holland, 1981

[12] E. Kröner, Stress space and strain space in continuum mechanics, Phys. Stat. Solids (b) 144, 39-44 (1987)

[13] E. Kröner, A Variational Principle in Nonlinear Dislocation Theory. In: Proc. 2nd Internat. Conf. Nonlinear Mechanics, ed. Chien Wei-zang, Peking University Press, Beijing, 1993, pp. 59-64

[14] E. Kröner, Dislocation theory as a physical field theory, In: Continuum Models and Discrete Systems, Proc. 8th Internat. Symp., Varna, Bulgaria, ed. K. Z. Markov, World Scientific, Singapore, 1996, pp. 522-537

[15] L. D. Landau and E. M. Lifshitz, Theory of Elasticity, Volume 7 of Course in Theoretical Physics, Pergamon Press, 1970

[16] J. E. Marsden and J. R. Hughes, Mathematical Foundations of Elasticity, Prentice Hall, Englewood Cliffs, New Jersey, 1983

[17] J. E. Marsden and T. S. Ratiu, Introduction to Mechanics and Symmetry, Springer-Verlag, New York, 1994

[18] G. A. Maugin, The method of virtual power in continuum mechanics: Application to coupled fields, Acta Mech. 35, 1-70 (1980)

[19] G. A. Maugin, The Thermodynamics of Plasticity and Fracture, Cambridge Texts in Applied Mathematics, Cambridge University Press, Cambridge, 1992

[20] W. Noll, Materially uniform simple bodies within homogeneities, Arch. Rational Mech. Anal. 27, 1-32 (1967)

[21] G. Schwarz, The Euclidean group in global models of continuum mechanics and the existence of a symmetric stress tensor, Rep. Math. Phys. 33, 397-412 (1993)

[22] G. Schwarz, Hodge Decomposition-A Method for Solving Boundary Value Problems, Lecture Notes in Mathematics No. 1607, Springer, Heidelberg, 1995

[23] M. Spivak, A Comprehensive Introduction to Differential Geometry, Vol. II, Publish or Perish, Boston, 1970 
[24] G. I. Taylor, The mechanism of plastic deformation of crystals, parts I and II, Proc. Roy. Soc. London A 145, 362-387, 388-404 (1934)

[25] C.-C. Wang, On the geometric structures of simple bodies. A mathematical foundation for the theory of continuous distributions of dislocations, Arch. Rational Mech. Anal. 27, 33-94 (1967)

[26] J. Wenzelburger, Ein globales differentialgeometrisches Modell dünner Schalen, Diplomarbeit Universität Karlsruhe, Karlsruhe, 1989

[27] J. Wenzelburger, Die Hodge-Zerlegung in der Kontinuumstheorie von Defekten, Dissertation Universität Mannheim, Verlag Shaker, Aachen, 1994

[28] J. Wenzelburger, A kinematic model for continuous distributions of dislocations, Journal of Geometry and Physics 24, 334-352 (1998) 